\title{
ESTRATÉGIAS DE TREINAMENTO ADMISSIONAL DA EQUIPE DE ENFERMAGEM DEUM CANCER CENTER DURANTE A PANDEMIA DO COVID-19
}

\author{
Maria das Graças S. Matsubara ${ }^{1}$ \\ Estela Ferreira da Silva ${ }^{2}$ \\ Regina Claudia Soares ${ }^{3}$ \\ Fernanda Cascapera ${ }^{4}$ \\ Daiane Arruda Saraiva ${ }^{5}$ \\ Benedito Aparecido da Silva ${ }^{6}$ \\ Eliza Costa Cijevschi ${ }^{7}$
}

\author{
https://orcid.org/0000-0003-2547-6788 \\ https://orcid.org/0000-0002-8586-593X \\ https://orcid.org/0000-0002-3575-1221 \\ https://orcid.org/0000-0003-2385-3965 \\ https://orcid.org/0000-0002-8128-9445 \\ https://orcid.org/0000-0003-4559-1468 \\ https://orcid.org/0000-0003-1676-9634
}

Objetivo: Descrever a elaboração da estratégia de ensino-aprendizagem adotada durante a pandemia do COVID-19, utilizando modelo híbrido (blended learning), tendo como proposta o treinamento de profissionais da Enfermagem recém admitidos, para atuar em Oncologia; apresentar os resultados da avaliação de aprendizagem dos programas educativos utilizando o modelo híbrido (blended learning), aplicados para os profissionais da Enfermagem recém admitidos; apresentar a percepção dos profissionais da Enfermagem recém admitidos sobre um programa educativo utilizando o modelo híbrido (blended learning). Método: Estudo descritivo tipo relato de experiência, realizado num hospital de alta complexidade em Oncologia da cidade de São Paulo, no periodo de março a maio de 2020, tendo como participantes, enfermeiros e técnicos em enfermagem recém-admitidos na instituição. Descrição da experiência: Ações educacionais foram realizadas durante a pandemia do COVID-19 e tiveram como referência a estratégia baseada no modelo hibrido (based learning) com a finalidade de treinar enfermeiros e técnicos de enfermagem para atuar na área de oncologia. Conclusão: O modelo hibrido demonstrou ter sido relevante no aprendizado dos alunos que demonstraram níveis elevados de satisfação após a intervenção proposta. Esta modelo demonstrou ser uma ferramenta importante e efetiva para o ensino no contexto da pandemia do COVID-19.

Descritores: Educação em Enfermagem; Serviço Hospitalar de Oncologia; Infecções por Coronavirus.

\section{ADMISSION TRAINING STRATEGIES OF THE NURSING TEAM OFA CANCER CENTER DURING THE COVID-19 PANDEMIC}

Objective: To describe the elaboration of a teaching-learning strategy adopted during the COVID-19 pandemic. The hybrid model (blended learning) was applied and it has a purpose of training nursing professionals newly admitted to work in the oncology field. To present the perception of nursing professionals newly admitted about an educational program using the hybrid model (blended learning). Methods: This is a descriptive study. An experience report was conducted in a hospital of high complexity which is reference in oncology in the city of São Paulo. Participants were composed of nurses and nursing technicians newly admitted in the analyzed institution. This research was conducted between March and April, 2020. Experience description: Educational actions were made during the COVID-19 pandemic. The hybrid model (based-learning) was applied as a reference for this strategy. This intervention had the purpose of training nurses and nursing technicians to work in the oncology field. Conclusion: The hybrid model has shown to be relevant in the student's learning. Students reported that they have achieved high levels of satisfaction after the proposed intervention. This model has demonstrated to be an important and effective tool for teaching in the context of COVID-19 pandemic.

Descriptors: Education in nursing; Hospital Service in Oncology; Coronavirus Infections.

ESTRATEGIAS DE CAPACITACIÓN DE ADMISIÓN DEL EQUIPO DE ENFERMERIA DE UN CENTRO DE CANCER DURANTE LA PANDEMIA DE COVID-19

Objetivo: Describir el desarrollo de la estrategia de la enseñanza y aprendizaje adoptada durante la pandemia COVID-19, utilizando un modelo híbrido (aprendizaje combinado), con la propuesta de capacitar a profesionales de enfermería recién ingresados para trabajar en oncología; presentar los resultados de la evaluación del aprendizaje de los programas educativos utilizando el modelo híbrido (aprendizaje combinado), aplicado a los profesionales de enfermería recién ingresados; presentar la percepción de los profesionales de enfermería recientemente admitidos acerca de un programa educativo que utiliza el modelo hibrido (aprendizaje combinado). Metodo: Este es un informe descriptivo de tipo de estudio de experiencia, realizado en un hospital de oncología de alta complejidad en la ciudad de São Paulo, de marzo a abril de 2020 , con participantes, enfermeras y técnicos de enfermería recientemente ingresados en la institución. Conclusión: Se realizaron acciones educativas durante la pandemia COVID-19. El modelo hibrido (aprendizaje combinado) se aplicó como referencia para esta estrategia. Esta intervención tenía el propósito de capacitar a enfermeras y técnicos de enfermería para trabajar en el campo de la oncología. Principales resultados alcanzados: El modelo hibrido ha demostrado ser relevante en el aprendizaje del alumno. Los estudiantes informaron que han alcanzado altos niveles de satisfacción después de la intervención propuesta. Este modelo ha demostrado ser una herramienta importante y efectiva para la enseñanza en el contexto de la pandemia de COVID-19.

Descriptores: Educación en Enfermeria; Servicio de oncología hospitalaria; Infecciones por coronavirus.

${ }^{1}$ A.C.Camargo Cancer Center, São Paulo, SP

Autor Correspondente: Maria das Graças da Silva Matsubara E-mail: maria.matsubara@accamargo.org.br Recebido: 10/5/20 Aceito:01/6/20 


\section{INTRODUÇÃO}

A pandemia gerada devido ao novo Coronavirus 2019 (COVID-19) é considerada um grave problema de saúde pública mundial, que requer ações emergenciais, no que tange, não só no controle da doença, mas também nos processos operacionais ${ }^{1,2}$.

Esta situação tem provocado o cancelamento de programas educativos e gerado novas concepções de aprendizagem nos motivando a rever as estratégias de ensino, implementando tecnologias diversificadas, fazendo com que o profissional faça parte da construção do seu conhecimento e por fim associando a prática, que é indispensável, somente quando necessário para evitar o contato físico dos profissionais ${ }^{3.4}$.

No Brasil, o primeiro caso foi registrado em 2 de março de 2020 , desde então houve aumento exponencial do vírus na população, sendo necessário a adoção de medidas para controlar a disseminação da doença ${ }^{5}$. Porém, as instituições de saúde precisaram rever o dimensionando de pessoal e providenciar a contratação de novos profissionais, o que exigiu a implantação de medidas emergenciais para o preparo destes que passarão por um duplo processo de estresse, envolvendo a crise de saúde pública com a disseminação do vírus, e uma nova rotina de trabalho.

Considerando que a falta de treinamento reflete diretamente, na produtividade, com consequente impacto na qualidade do processo assistencial, a proposta do treinamento admissional na área da Enfermagem, visa preparar os profissionais da equipe para uma assistência baseada em protocolos, processos e diretrizes, alinhado ao propósito, visão e valores da instituição. Trata-se de processo organizado, para propiciar o conhecimento e desenvolvimento de habilidades para alcançar determinada competência por meio da experiência prática orientada e feedback regular'b. Ou seja, este é um recurso essencial para promover a adaptação dos novos profissionais à instituição, minimizando a variabilidade no processo de cuidar, favorecendo a propagação das normas, rotinas e protocolos na prestação de uma assistência de qualidade e segurab.

Portanto, para que se estabeleça um patamar de qualidade, planos educacionais devem ser empreendidos, pois isto reflete diretamente na prática cotidiana desses profissionais, que precisam ter alinhado, teoria e prática ${ }^{7}$.

Quando trata se de qualificação para o trabalho na Oncologia emerge outras preocupações, que envolve o despreparo do profissional, com a lacuna deixada pela formação e a complexidade tecnológica contida na especialidade ${ }^{8}$. Essa demanda impulsiona e determina a busca de eficiência nos processos de treinamento, com múltiplas estratégias educacionais para capacitar com o propósito de adequá-los às exigências do mundo do trabalho ${ }^{7}$

As instituições que prestam assistência para pacientes com câncer devem promover atividades educativas eficientes para os profissionais de enfermagem que cuidam desses pacientes e suas familias, técnica e cientificamente ${ }^{7}$.

Frente à esta pandemia, a instituição também deve ofertar programas de treinamentos, destinados a aprimorar o conhecimento sobre o COVID-19, pois isto mantem atitudes otimistas e práticas apropriadas no cuidado ${ }^{2}$. Para atender tais necessidades, a Educação à Distância (EaD) surge como uma opção de adaptação do programa de treinamento admissional presencial, tendo em vista a exigência atual de distanciamento físico?.

A EaD refere-se ao processo de ensino-aprendizagem, onde o estudante e o instrutor estão fisicamente separados, mediados por sistemas de telecomunicações interativos, utilizados para conectar estudante, recursos e instrutores 9.10

Nesse cenário, o ensino híbrido ou blended learning, no qual são agregadas diversas formas de ensino, que inclui encontros presenciais e digital, as quais estabelecem a colaboração, o aprender juntos e a personalização, os percursos individuais, pautados na necessidade, com a participação ativa do estudante, que pode ser uma das propostas pedagógicas para atender as demandas do século $X X \mathrm{I}^{10}$.

Numa pandemia, as boas práticas educacionais com alinhamento construtivo e articulado, acolhimento do ingressante, valorizando o conhecimento prévio, seu potencial individual, favorece a integração com o coletivo e auxilia na compreensão sobre a mudança, com consequente redução da ansiedade ${ }^{6,9}$.

Considerando a demanda de capacitar novos colaboradores, frente à atual pandemia e seguindo as recomendações sanitárias, este estudo emerge com o objetivo de descrever as estratégias de ensino-aprendizagem adotadas durante a pandemia do COVID-19, utilizando modelo hibrido (blended learning), tendo como proposta a capacitação de profissionais da Enfermagem recém-admitidos, para atuar em Oncologia.

\section{OBJETIVOS DA EXPERIÊNCIA}

Descrever a elaboração da estratégia de ensino-aprendizagem adotada durante a pandemia do COVID-19, utilizando modelo hibrido (blended learning), tendo como proposta o treinamento de profissionais da Enfermagem recém admitidos, para atuar em Oncologia; apresentar os resultados da avaliação de aprendizagem do programa educativos utilizando o modelo hibrido (blended learning), aplicados para os profissionais da Enfermagem recém admitidos; apresentar a percepção dos profissionais da Enfermagem recém admiti- 
dos sobre um programa educativo utilizando o modelo híbrido (blended learning).

\section{MÉTODO}

\section{Tipo de estudo}

Trata-se de estudo descritivo tipo relato de experiência.

\section{Sujeitos envolvidos na experiência}

Foram participantes, os dados contidos no arquivo institucional referentes a 11 enfermeiros e 70 técnicos em Enfermagem recém-admitidos numa instituição oncológica de alta complexidade.

\section{Cenário do estudo}

Realizado na área de Ensino, Departamento de Educação Continuada (EC) de um Cancer Center da cidade de São Paulo. É uma instituição privada de cunho filantrópico, de assistência, ensino e pesquisa em câncer.

\section{Periodo de realização da experiência}

Período de março a maio de 2020

\section{Aspectos éticos}

Estudo foi submetido e apreciado pelo Comitê de Ética em Pesquisa da Fundação Antônio Prudente, sob o número de protocolo 4.015.983. A pesquisa dispensa o Termo de Consentimento Livre e Esclarecido, uma vez que a coleta de dados foi realizada mediante o uso do informações disponíveis nos arquivos da área de Ensino.

\section{DESCRIÇÃO DA EXPERIÊNCIA}

Elaborado programa educativo com carga horária distinta para enfermeiro e técnico, sendo um total de 24 horas para técnico em enfermagem, destas, 18 horas online e 6 horas presencial; total de 30 horas para o enfermeiro, sendo 19 horas online e 11 horas presencial.

O plano de curso teve como proposta desenvolver os objetivos com base na Taxonomia de Bloom, ancorado nos dominios cognitivos, psicomotores e afetivos ${ }^{(8)}$. Para a definição da estratégia quanto a proposta híbrida, os objetos de aprendizagem (OA) foram construídos com base nas tipologias de conteúdo factual, conceitual, procedimental e atitudinal( ${ }^{(8)}$.

A proposta pedagógica relacionada à metodologia de ensino, foi estruturada utilizando quiz e fóruns, como instrumentos de avaliação de aprendizagem, tendo como base os conteúdos disponíveis no Ambiente Virtual de Aprendizagem (AVA). Além disso foi aplicada avaliação de reação, para obter a percepção dos profissionais sobre o programa educativo admissional.

O programa educativo online foi ofertado com apoio da plataforma MOODLE (Modular Object-Oriented Dynamic Learning Environment), que é um software livre de suporte à aprendizagem, executado num ambiente virtual(8,11). Também foram utilizados outros recursos tecnológicos, como o WhatsApp® e ferramentas da Microsoft $®$, como o formulário forms para avaliação do programa prático e o Teams®, para interação síncrona. O Teams é um hub digital que reúne conversas, conteúdos, tarefas e aplicativos em um só lugar ${ }^{(12)}$

A instituição disponibilizou tablets para uso na área do Ensino, para profissionais que não tivessem recursos tecnológicos e ou internet, para que pudessem acessar ao AVA e realizar o treinamento. Porém, nenhum profissional teve dificuldade com o acesso à internet ou recurso tecnológico. O grupo de dúvidas do WhatsApp® foi organizado no celular corporativo da área de Ensino e consistia num canal para dúvidas técnicas sobre o AVA.

As admissões ocorrem a cada quinzena, o que suscita a necessidade de treinamento convergente com esta fase. Portanto, o programa educativo aconteceu em três períodos: período um $(\mathrm{Pl})$ refere-se à primeira quinzena de abril, periodo 2 (P2), segunda quinzena de abril e período 3 (P3) primeira quinzena de maio.

Ao término de cada período foram realizados encontros com os enfermeiros da EC, com o objetivo de identificar oportunidades de melhorias.

\section{PRINCIPAIS RESULTADOS ALCANÇADOS}

A apresentação dos resultados considerou-se: a) descrição do processo de estruturação do programa educativo com referenciais teóricos utilizados; b) avaliação de aprendizagem contendo variáveis quantitativas, valores das médias e medianas, desvios padrão, mínimo e máximo relacionado a categoria profissional; b) percentual de favorabilidade quanto aos conceitos adotados para a avaliação de reação sobre os programas educativos.

As oportunidades de melhorias identificadas consistiram em revisões e ajustes dos OA, reorganização dos conteúdos quanto aos módulos disponiveis no AVA entre o Pl e P2. No P3 foi adotado momentos síncronos, com uso da plataforma Teams® e excluído a comunicação via WhatsApp®

A decisão por não manter o grupo WhatsApp ${ }^{\circledR}$ foi tomada devido a intensificação da discussão relacionada ao conteúdo técnico, que deveria ser feita no AVA e conversas particulares.

No início de cada programa foram realizados encontros presenciais, tendo como finalidade apresentar o programa educativo, cadastrar a senha e orientar sobre a navegação na plataforma. A efetividade no processo de ensino-aprendizagem online está intrinsecamente associada à introdução à tecnologia, como acessar os recursos disponíveis no AVA, o que direciona os estudantes para uma mudança de expectativa quanto a aprender e tirar maior proveito de um ambiente 
de aprendizagem via web $b^{(13,14)}$.

1. Estratégia de ensino-aprendizagem utilizando modelo híbrido (blended learning)

Participaram do programa 81 profissionais de Enferma- gem, sendo 11 enfermeiros e 70 técnicos em enfermagem.

A tabela 1 mostra a relação de profissionais participantes do programa educativo distribuídos por periodo e categoria profissional.

Tabela 1 - Relação de profissionais participantes no programa educativo por período e categoria profissional

\begin{tabular}{|c|c|c|c|c|}
\hline Categoria profissional & P1 & P2 & P3 & Total \\
\hline Enfermeiro & 10 & 1 & 0 & 11 \\
\hline Técnico Enfermagem & 19 & 29 & 22 & 70 \\
\hline Total & 29 & 30 & 22 & 81 \\
\hline
\end{tabular}

\subsection{Programa educativo online}

Utilizada a plataforma MOODLE para disponibilização dos conteúdos e avaliação do programa. Nesta etapa foram denominados como professor-tutor aquele que, além de produzir o conteúdo, também assumiu o papel de tutor durante a realização do treinamento. $O$ suporte técnico foi oferecido por uma equipe responsável por questões técnicas referentes à plataforma, solucionando eventuais problemas de operação, manutenção e configuração dos equipamentos, realizando instalações e/ou fornecendo suporte aos softwares necessários.

Foram elaborados e disponibilizados 76 OA, incluindo ví- deos, infográficos, e-book, Scorm, jogos educativos, fórum e quizzes.

Com base nas oportunidades de melhoria identificadas foram organizados no P3 encontros síncronos pela plataforma Teams®. Esses momentos aconteceram nos três primeiros dias de treinamento, sempre às $7 \mathrm{~h}$ e $12 \mathrm{~h}$. O primeiro momento tinha o objetivo de direcionar os estudos, já no segundo, a proposta foi de revisar o conteúdo. Ambas ocasiões tiveram a mediação de dois a três enfermeiros da EC

Os conteúdos foram organizados em oito módulos, conforme descrito na figura 1 .

Figura 1 - Programa de treinamento admissional online para profissionais da Enfermagem recém-admitidos

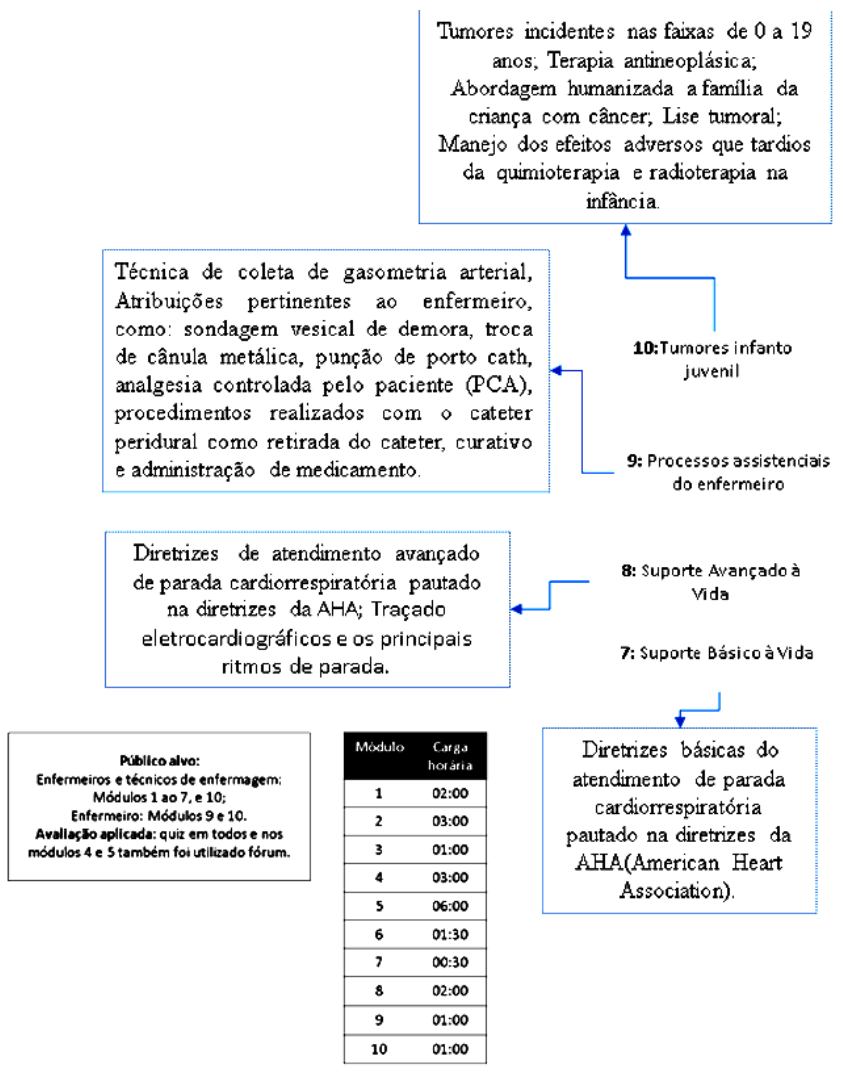

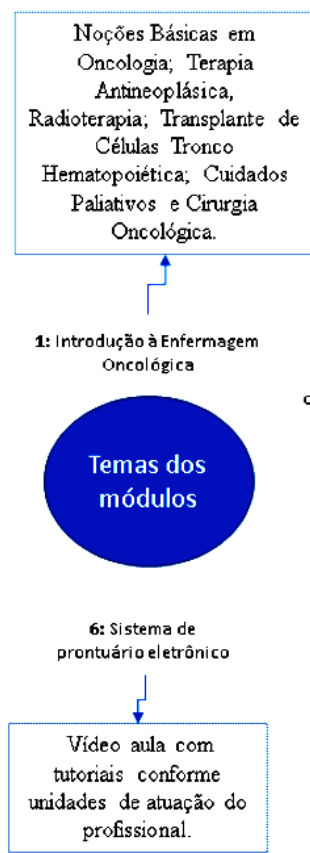

profissional. 


\subsection{Programa educativo presencial}

O programa presencial foi composto por conteúdos relacionados ao domínio psicomotor, tendo como objetivo desenvolver habilidade mínima para atuação. Teve como proposta a utilização da estratégia com o formato de oficinas, organizadas em cinco estações, mediadas por um enfermeiro da EC. Cada oficina foi realizada com no máximo cinco participantes em sala, o que propiciava um distanciamento físico seguro entre os participantes e o mediador.

O enfermeiro participou de aula prática sobre punção de cateter totalmente implantado, e treinamento on the job (acontece dentro do ambiente de trabalho com a supervisão de um multiplicador) no ambulatório de Quimioterapia, acompanhado por um multiplicador da área.

Os conteúdos foram organizados em cinco estações, conforme descrito na figura 2.

Figura 2 - Programa de treinamento presencial para profissionais da Enfermagem recém-admitidos

\begin{tabular}{|c|c|c|c|c|}
\hline Oficina $1^{*}$ & Oficina $2^{*}$ & Oficina $3^{*}$ & Oficina $4^{*}$ & Oficina $5 *$ \\
\hline $\begin{array}{l}\text { Bomba Parenteral } \\
\text { Protocolo de Dor } \\
\text { Prática de TEV }\end{array}$ & $\begin{array}{l}\text { Bomba Enteral } \\
\text { Protocolo de hipoglicemia } \\
\text { Manuseio de Insulinas }\end{array}$ & $\begin{array}{l}\text { Drenos } \\
\text { Estomas }\end{array}$ & Terapia Intravenosa & Sistema Tasy \\
\hline $\begin{array}{c}\text { - Exposição e programação da } \\
\text { bomba de infusão para } \\
\text { medicamentos; } \\
\text { - Preparo de ATB e instalação; } \\
\text { - Preparo de soro e instalação; } \\
\text { - Validade dos equipos; } \\
\text { - Protocolo de avaliação da dor e } \\
\text { interpretação das escalas BPS e } \\
\text { Categórica Numérica. } \\
\text { - Mensuração e colocação das } \\
\text { meias compressivas } \\
\text { antiembólicas. }\end{array}$ & $\begin{array}{l}\text { Exposição e programação da } \\
\text { bomba de infusão para dieta por } \\
\text { sonda. } \\
\text { - Tipos de sondas padronizadas na } \\
\text { instituição: SNE, jejunostomia e } \\
\text { gastrostomia; } \\
\text { - Protocolo de desobstrução; } \\
\text { - Material padronizado para fixação } \\
\text { das sondas; } \\
\text { - Cuidados com risco de lesão por } \\
\text { pressão não clássica; } \\
\text { - Procedimento de administração } \\
\text { de medicação pela sonda. } \\
\text { - Tipos de insulina: Início; Pico e } \\
\text { duração; Seringa de insulina; } \\
\text { - Manuseio da agulha auto shield } \\
\text { (caneta) e caneta de insulina; } \\
\text { - Manuseio do monitor de glicemia. }\end{array}$ & $\begin{array}{l}\text { - Indicações, procedimentos de } \\
\text { cada dreno (sucção, } \\
\text { capilaridade e gravitacional); } \\
\text { Curativo; Fixação (meso); } \\
\text { - Indicações, tipos e } \\
\text { procedimentos dos estomas: } \\
\text { intestinais, urinários } \\
\text { respiratórios e gástricos. }\end{array}$ & $\begin{array}{l}\text { - Indicações, procedimentos e } \\
\text { tipos de cateteres periférico e } \\
\text { central. } \\
\text { - Demonstração da técnica de } \\
\text { turbilhonamento; } \\
\text { Demonstração da técnica de } \\
\text { punção e aplicação de película } \\
\text { transparente. Procedimento e } \\
\text { orientação de prevenção de } \\
\text { flebite e extravasamento das } \\
\text { medicações, uso de EPIs }\end{array}$ & $\begin{array}{l}\text { - Apresentação dos tutoriais } \\
\text { Tasy. } \\
\text { Prática exclusiva do } \\
\text { enfermeiro }\end{array}$ \\
\hline
\end{tabular}

2. Resultados da avaliação de aprendizagem do programa educativo utilizando o modelo híbrido (blended learning)

A avaliação de aprendizagem foi realizada por módulo, com uso de quiz e fórum. Os quizzes continham de 2 a 12 questões, de acordo com a complexidade do tema. Os fóruns de discussão foram organizados com perguntas norteadoras relacionada aos conteúdos com objetivo afetivo, tais como, processo medicamentoso e rotinas organi- zacionais requeridas.

Nos módulos com mais de uma atividade avaliativa foi considerada a média de todas as notas, sendo que, cada atividade vale de $\mathrm{O}$ a 10 pontos. A realização das atividades não teve limitação de tentativas. e foi considerado a maior nota para a média final.

A tabela 2 apresenta os resultados da avaliação de aprendizagem de acordo com a categoria profissional. 
Tabela 2 - Resultados da avaliação de aprendizagem de acordo com a categoria profissional

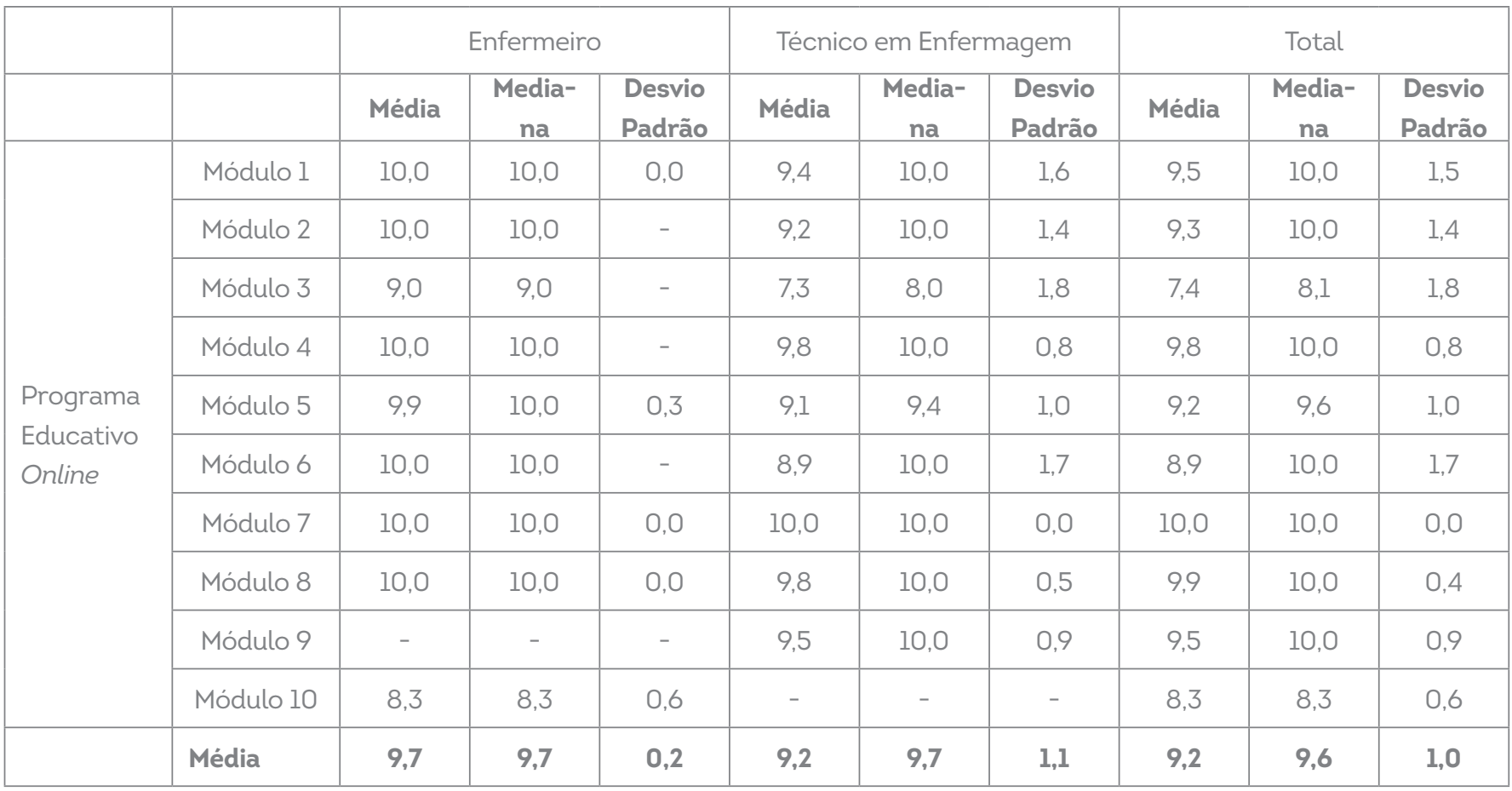

Observa-se média acima de 9 para ambas as categorias profissionais.

\section{Percepção dos colaboradores nos programas educa- tivos utilizando o modelo híbrido (blended learning).}

Em relação a avaliação sobre a percepção dos participantes quanto ao programa educativo foram aplicados questionários específicos com conceitos distintos.

\subsection{Programa educativo online}

Para avaliação do programa online foi utilizado instrumento próprio do MOODLE, que tem conceitos variando de 0 a 10. Porém, os itens foram definidos pela área de EC, com base na intencionalidade de obter respostas fidedignas às propostas educativas. Para a análise da per- cepção apoiou-se na metodologia Net Promoter Score (NPS®), a qual considera pontuação de 9 a 10 como promotor, sinalizando enriquecimento da experiência, entre 7 e 8 passivamente satisfeitos ou neutros e de 0 a 6 detratores, indicam uma experiência ruim.

O cálculo é realizado utilizando a seguinte fórmula, percentual de promotores menos o percentual de detratores. A partir deste cálculo obtêm-se os valores do NPS, que apontam a posição do objeto avaliado, classificado da seguinte forma: 75\% indica excelência, 50 a 74\%, sinaliza qualidade, 0\% e $49 \%$ recomenda aperfeiçoamento e menor que $49 \%$ indica situação crítica $^{(15)}$

Os resultados sobre a percepção dos participantes no programa educativo online estão apresentados na tabela 3.

Tabela 3 - Resultados da percepção dos participantes no programa educativo online

\begin{tabular}{|c|c|c|c|c|c|c|c|c|c|c|c|c|c|c|c|c|}
\hline \multirow[b]{2}{*}{ Critérios avaliados } & \multicolumn{4}{|c|}{ P1 } & \multicolumn{4}{|c|}{ P2 } & \multicolumn{4}{|c|}{ P3 } & \multicolumn{4}{|c|}{ Consolidado } \\
\hline & $\mathbf{N}$ & $\mathbf{P}$ & D & NPS & $\mathbf{N}$ & $\mathbf{P}$ & D & NPS & $\mathbf{N}$ & $\mathbf{P}$ & D & NPS & $\mathbf{N}$ & $\mathbf{P}$ & D & NPS \\
\hline $\begin{array}{l}\text { O formato do treina- } \\
\text { mento (metodologia) } \\
\text { foi atrativo e esclare- } \\
\text { cedor? }\end{array}$ & 6 & 3 & 0 & 50 & 17 & 14 & 1 & 76,5 & 17 & 12 & 1 & 64,7 & 40 & 29 & 2 & 67,5 \\
\hline $\begin{array}{l}\text { O conteúdo programá- } \\
\text { tico foi de fácil com- } \\
\text { preensão? }\end{array}$ & 6 & 5 & 0 & 83,3 & 17 & 13 & 2 & 64,7 & 17 & 11 & 0 & 64,7 & 40 & 29 & 2 & 67,5 \\
\hline
\end{tabular}




\begin{tabular}{|c|c|c|c|c|c|c|c|c|c|c|c|c|c|c|c|c|}
\hline $\begin{array}{l}\text { Como você avalia o } \\
\text { tempo de duração do } \\
\text { treinamento? }\end{array}$ & 6 & 3 & 2 & 16,7 & 17 & 12 & 3 & 52,9 & 17 & 6 & 5 & 5,9 & 40 & 21 & 10 & 27,5 \\
\hline $\begin{array}{l}\text { O quanto você se sen- } \\
\text { tiu acolhido ao realizar } \\
\text { a integração Enferma- } \\
\text { gem }\end{array}$ & 6 & 5 & 0 & 83,3 & 17 & 16 & 1 & 88,2 & 17 & 15 & 0 & 88,2 & 40 & 36 & 1 & 87,5 \\
\hline $\begin{array}{l}\text { Como você avalia a } \\
\text { comunicação e divul- } \\
\text { gação prévia do treina- } \\
\text { mento? }\end{array}$ & 6 & 2 & 0 & 33,3 & 17 & 15 & 0 & 88,2 & 17 & 15 & 0 & 88,2 & 40 & 32 & 0 & 80 \\
\hline $\begin{array}{l}\text { Ao acessar o AVA você } \\
\text { recebeu o suporte ne- } \\
\text { cessário para navegar } \\
\text { sem dificuldades? }\end{array}$ & 6 & 4 & 1 & 50 & 17 & 15 & 1 & 82,4 & 17 & 13 & 0 & 76,5 & 40 & 32 & 2 & 75 \\
\hline $\begin{array}{l}\text { De maneira geral, } \\
\text { como você avalia o } \\
\text { treinamento? }\end{array}$ & 6 & 3 & 1 & 33,3 & 17 & 13 & 1 & 70,6 & 17 & 11 & 0 & 64,7 & 40 & 27 & 2 & 62,5 \\
\hline Média & & & & 50 & & & & 74,8 & & & & 64,7 & & & & 66,8 \\
\hline
\end{tabular}

Legenda: $N=$ =neutro, $D=$ detrator, $P=$ promotor

Tendo como referência o NPS, observa-se que a nota global dos participantes manteve um nivel elevado de favorabilidade, com melhor escore no P2.

\subsection{Programa educativo presencial}

A avaliação das oficinas foi realizada por meio do formulário eletrônico da Microsoft Forms®, contendo itens rela- cionados a proposta pedagógica. Foram adotados conceitos referentes a escala de likert de 5 pontos, sendo 0 para ruim e 5 para ótimo.

Os resultados sobre a percepção dos participantes no programa educativo presencial contemplando os três períodos estão apresentados na tabela 4.

Tabela 4 - Resultados da percepção dos participantes no programa educativo presencial

\begin{tabular}{|l|c|c|c|c|c|c|c|c|c|}
\hline & \multicolumn{2}{|c|}{ Ótimo } & \multicolumn{2}{c|}{ Bom } & \multicolumn{2}{c|}{ Regular } & \multicolumn{2}{c|}{ Ruim } & Total \\
\hline \multicolumn{1}{|c|}{ Itens avaliados } & N & $\%$ & $\mathbf{N}$ & $\%$ & $\mathbf{N}$ & $\%$ & $\mathbf{N}$ & $\%$ & N \\
\hline $\begin{array}{l}\text { Oficina: manuseio da bomba de } \\
\text { infusão enteral; glicemia capilar; } \\
\text { caneta de insulina; tipos de son- } \\
\text { das }\end{array}$ & 47 & 88,7 & 6 & 11,3 & 0 & 0,0 & 0 & 0,0 & 53 \\
\hline $\begin{array}{l}\text { Oficina: manuseio bomba de } \\
\text { infusão parenteral; colocação de } \\
\text { meias antiembólicas; protocolo } \\
\text { de dor }\end{array}$ & 40 & 75,5 & 13 & 24,5 & 0 & 0,0 & 0 & 0,0 & 53 \\
\hline $\begin{array}{l}\text { Oficina: estomas respiratório, } \\
\text { urinário e intestinal; tipos de } \\
\text { drenos indicação e os cuidados } \\
\text { de enfermagem }\end{array}$ & 41 & 77,3 & 12 & 22,7 & 0 & 0,0 & 0 & 0,0 & 53 \\
\hline
\end{tabular}




\begin{tabular}{|l|c|c|c|c|c|c|c|c|c|}
\hline $\begin{array}{l}\text { Oficina: PEP com acesso à pla- } \\
\text { taforma Tasy® }\end{array}$ & 31 & 58,4 & 18 & 34,0 & 4 & 7,6 & 0 & 0,0 & 53 \\
\hline $\begin{array}{l}\text { Oficina: terapia intravenosa; } \\
\text { tipos de cateteres indicação e } \\
\text { os cuidados de enfermagem }\end{array}$ & 33 & 75,0 & 10 & 22,7 & 1 & 2,3 & 0 & 0,0 & 44 \\
\hline $\begin{array}{l}\text { De uma forma geral como você } \\
\text { avalia a apresentação dos con- } \\
\text { teúdos nas oficinas }\end{array}$ & 38 & 71,7 & 15 & 28,3 & 0 & 0,0 & 0 & 0,0 & 53 \\
\hline Total & $\mathbf{2 3 0}$ & $\mathbf{7 4 , 4}$ & $\mathbf{7 4}$ & $\mathbf{2 4 , 0}$ & $\mathbf{5}$ & $\mathbf{1 , 6}$ & $\mathbf{0}$ & $\mathbf{0 , 0}$ & $\mathbf{3 0 9}$ \\
\hline
\end{tabular}

Observa-se que de forma geral o programa prático recebeu conceito ótimo de $74,4 \%$.

\section{Limitações da experiência}

Tempo limitado para organização do programa educativo com vistas à educação permanente numa área de alta complexidade, que possui déficit relacionado às instituições formadoras; cultura relacionada a programas educativos online, que esbarra na fluência digital e planejamento para estudos;

Disponibilidade de internet com velocidade adequada para acessar OA como vídeos e SCORM.

\section{Contribuições para a prática}

Propiciar um modelo instrucional de programa educativo para profissionais da Enfermagem recém-admitidos, utilizando a estratégia hibrida (blended learning), a fim de mitigar riscos assistenciais e de contaminação, uma vez que tal proposta propicia o cumprimento de recomendação do Ministério da Saúde e OMS, durante a pandemia do COVID-19, com o distanciamento físico.

Contribuições dos Autores: Maria das Graças Silva Matsubara, Estela Ferreira da Silva, Fernanda Cascapera: concepção e desenho do estudo; Daiane Arruda Saraiva, Regina
Claudia Soares, Benedito Aparecido da Silva, Eliza Costa Cijevschi: coleta, análise e interpretação dos dados; Maria das Graças Silva Matsubara, Estela Ferreira da Silva, Fernanda Cascapera, Daiane Arruda Saraiva, Regina Claudia Soares, Benedito Aparecido da Silva, Eliza Costa Cijevschi: redação e/ou revisão crítica do manuscrito; Maria das Graças Silva Matsubara, Estela Ferreira da Silva, Regina Claudia Soares: aprovação da versão final a ser publicada;

\section{CONCLUSÃO}

O presente estudo descreveu a adequação do ensino e de uma instituição especialista em câncer para o treinamento de profissionais recém-admitidos, frente a situações novas, como o cuidado ao paciente com câncer e a presente pandemia. Torna-se um desafio a elaboração de treinamentos $\mathrm{EaD}$ em uma área na qual busca-se a interação entre os profissionais para o desenvolvimento cognitivo e afetivo, tendo a necessidade de treinar habilidades psicomotoras.

Sabe-se que são necessários novos estudos, quando à esta nova realidade para a área da saúde, espera-se que tal relato de experiência possa contribuir para o desenvolvimento de novas estratégias de ensino.

\section{REFERÊNCIAS}

1. Han X, Wang X, Zhang M, Wang X. Using Social Media to Mine and Analyze Public Opinion Related to COVID-19 in China. Int. J. Environ. Res. Public Health 2020 Abr; 17 (8): 2788. [acesso em 26 abr 2020]. Disponivel em: https://www. mdpi.com/1660-4601/17/8/2788.

2. Zhong BL, Luo W, Li HM, Zhang QQ, Liu XG, Li WT, et al. Knowledge, attitudes, and practices towards COVID-19 among Chinese residents during the rapid rise period of the COVID-19 outbreak: a quick online cross-sectional survey. Int. J. Biol. Sci. 2020; 16(10): 1745-52. [acesso em 26 abr 2020]. Disponivel em: https://www.ncbi.nlm.nih.gov/pmc/ articles/PMC7098034/.

3. Teixeira E, Vale EG. Desafios para reinventar o ensino e perspectivas para o curso de graduação em enfermagem. Enfermagem em Foco 2011; 1(2): 55-8. [acesso em 26 mai 2020]. Disponivel em: <http://revista.cofen.gov.br/index.php/ enfermagem/article/view/15/16>. 
4. Lipomi DJ. Video for Active and Remote Learning. Trends in Chemistry 2020; 6(2): 483-85. [acesso em 22 abr 2020]. Disponivel em: https://www.sciencedirect.com/science/article/pii/S2589597420300769.

5. Rafael RMR, Neto M, Carvalho MMB, David HMSL, Acioli S, Faria MGA. Epidemiologia, políticas públicas e Covid-19: o que esperar no Brasil. Rev enferm UERJ 2020; 28:e49570. [acesso em 22 abr 2020]. Disponivel em: https://www.epublicacoes.uerj.br/index.php/enfermagemuerj/article/view/49570.

6. Bucchi SM, Mira VL, Otrenti E, Ciampone MHT. Enfermeiro instrutor no processo de treinamento admissional do enfermeiro em unidade de terapia intensiva. Acta Paul Enferm 2011; 24(3): 381-7. [acesso em 20 abr 2020 ]. Disponivel em: https://www.scielo.br/scielo.php?script=sci_arttextEpid=S0103-21002011000300012.

7. Matsubara MGS, De Domenico EBL. Virtual Learning Environment in Continuing Education for Nursing in Oncology: an Experimental Study. J Canc Educ 2016 31:804-10. [acesso em 26 abr 2020]. Disponivel em: https://pubmed.ncbi. nlm.nih.gov/26224242/.

8. Matsubara MGS, De Domenico EBL. Building a Virtual Learning Environment for Distance Education in Nursing Oncology. Int Arch Nurs Health Care 2016; 2:049. [acesso em 26 abr 2020]. Disponivel em: https://clinmedjournals. org/articles/ianhc/international-archives-of-nursing-and-health-care-ianhc-2-049.php?jid=ianhc.

9. Pather N, Blyth P, Chapman JA, Dayal MR, Flack NAMS, Fogg QA, et al. Forced Disruption of Anatomy Education in Australia and New Zealand: An Acute Response to the Covid-19 Pandemic. Anat Sci Educ. 2020 Abr; 1-14. [acesso em 20 abr 2020]. Disponivel em https://anatomypubs.onlinelibrary.wiley.com/doi/epdf/10.1002/ase.1968.

10. Rolindo JMR, Reis MA, Almeida FR, Aranha TC, Melo JM, Correia SF, et al. Modelo hibrido: possibilidade de ensino no século XXI. Braz. J. of Develop 2019; 5(9): 14262-79. [acesso em 20 abr 2020]. Disponivel em: http://www. brazilianjournals.com/index.php/BRJD/article/view/3085/3004.

11. Martins CLM, Queluci GC. Situação Problema Como Estratégia de Ensino a Distância Sobre Prevenção de Lesão por Pressão. Enfermagem em Foco 2019; 6(10): 99-104. [acesso em 26 mai 2020]. Disponivel em: http://revista. cofen.gov.br/index.php/enfermagem/article/view/2697/657.

12. Microsoft. Introdução ao Microsoft Teams para aprendizado remoto [Internet]. 2020. [acesso em 22 abr 2020 ]. Disponivel em : https://docs.microsoft.com/pt-br/microsoftteams/remote-learning-edu

13. Pintz C, Posey L. Preparing students for graduate study: an eLearning approach. Nurse Educ Today 2013; 7(33): 3337. [acesso em 22 abr 2020]. Disponivel em: https://www.sciencedirect.com/science/article/pii/S0260691712003930

14. Horiuchi S, Yaju Y, Koyo M, Sakyo Y, Nakayama K. Evaluation of a web-based graduate continuing nursing education program in Japan: A randomized controlled trial. Nurse Educ Today. 2009; 29(2): 140-9. [acesso em 24 abr 2020$].$ Disponivel em: https://www.sciencedirect.com/science/article/pii/S0260691708001184?via\%3Dihub[acesso em 24 abr 2020]. Disponivel em: https://www.researchgate.net/publication/328305049_HOW_NET_PROMOTER_ SCORE_RELATES_TO_ORGANIZATIONAL_GROWTH 